\title{
Long intergenic non-coding RNA 1939 inhibits proliferation and migration of human renal cell carcinoma (RCC) cells by down-regulation of miR- 154
}

\section{Rongyuan Zhang}

First Affiliated Hospital of Soochow University

\section{Yuhua Huang}

First Affiliated Hospital of Soochow University

\section{Baocai Xu}

Jining No 1 People's Hospital

\section{Chuan LV}

Jining No 1 People's Hospital

Jianquan Hou

First Affiliated Hospital of Soochow University

Weijie Zhang ( $\nabla$ zhangwj0166@163.com )

First Affiliated Hospital of Soochow University https://orcid.org/0000-0002-6881-9165

\section{Research article}

Keywords: Renal Carcinoma, LINC01939, miR-154, Proliferation, Migration

Posted Date: June 24th, 2019

DOI: https://doi.org/10.21203/rs.2.10547/v1

License: (c) (i) This work is licensed under a Creative Commons Attribution 4.0 International License.

Read Full License 


\section{Abstract}

Background: Renal carcinoma (RCC) is widely accepted as a malignant tumor of urinary system. Long intergenic non-coding RNA 1939 (LINC01939) is a novel IncRNA which was found to be down-regulated in RCC. Thus, we set out to explore the effect and regulation mechanism of LINC01939 in RCC. Methods: LINC01939 and miR-154 in RCC tissues and cell lines were detected using qRT-PCR assay. Cell counting kit-8 (CCK-8) assays was exploited to examine cell viability. Flow cytometric analysis was conducted to examine apoptosis. Cell mobility was valued through wound healing assays. Western blotting was applied for examination of proteins related to proliferation, apoptosis, migration and Wnt/ $\beta$ catenin/Notch. Results: LINC01939 was down-regulated in RCC tissues. LINC01939 overexpression impeded proliferation and migration, and induced apoptosis. Further study found that the overexpression of LINC01939 strongly suppressed miR-154 expression. Then, the inhibiting effect of overexpressed LINC01939 on proliferation and mobility and the promoting role of LINC01939 in apoptosis were abolished by the combination of miR-154 mimic. Finally, we found that the overexpressed LINC01939 inactivated Wnt/ $\beta$-catenin and Notch through suppressing miR-154. Conclusion: The up-regulation of LINC01939 inhibited proliferation and migration of RCC cells by down-regulating miR-154.

\section{Introduction}

As one of the 10 most common malignant obesity tumors, renal cell carcinoma (RCC) is a common chemotherapeutic drug-resistant disease. The global annual incidence of RCC is about 20,000 cases, with 102,000 deaths [ 1]. Among them, clear cell renal cell carcinoma (ccRCC) is the most common type, accounting for $80-90 \%$, with the characteristics of local invasion, distant metastasis and high lethal rate [ 2]. Thus, the prognosis of patients with ccRCC is worse and the incidence of ccRCC is gradually increasing with a mortality rate about $40 \%$ [3]. At present, the main treatment of RCC is surgical treatment. However, even after early radical surgery, there are still many tumors recurrence and metastasis cases. Thus, it is of great significance to investigate the molecular mechanisms of RCC with multi-gene and multi-level diagnostic and therapeutic tools, aiming to get in-depth understanding of RCC.

Long non-coding RNA (IncRNA), defined as RNAs with no protein coding potential, is longer than $200 \mathrm{nt}$. LncRNA was initially thought to be merely transcriptional noise, which has no biological function [4]. But due to the growing depth of sequencing technology, more and more new IncRNAs have been found to be involved in various physiological and pathological processes [5]. Studies have shown that a number of IncRNAs are abnormally expressed in many tumors and may be involved in multiple processes of tumorigenesis and development, including cell differentiation and proliferation, cell cycle regulation, invasion and metastasis [ 6-8]. Thus, IncRNAs have been regarded as new biomarkers in various cancers including RCC. Recently, Chen et al. revealed that long intergenic non-coding RNA 1939 (LINC01939) is down-regulated in gastric cancer tissues and is associated with clinical stage of gastric cancer patients [ 9]. However, the biological function in RCC has never been explored as well as the underlying mechanisms. 
microRNAs (miRNAs or miRs) are recognized as the small non-coding RNAs essentially with a length of 18-25 nt, which are widely distributed in eukaryotic organisms. They are mainly responsible for regulating gene and protein expression and participating in many physiological and pathological processes, for instance, cell growth, differentiation and apoptosis [10]. These single-stranded mature miRNAs mediate protein coding by binding to $3^{\prime}$-untranslated region ( $3^{\prime}$-UTR) homologous sequence of their target gene mRNA [11]. miRNAs are very important regulator in urinary tumors and the expression and function of miRNAs can be observed in most urinary tumors. For example, up-regulation of miR-21 and miR-221 has been observed in tumor specimens relative to normal tissue, which is associated with the pathological status of patients [12]. Besides, Lin et al. found that miR-154 upregulation impedes apoptosis process and facilitates proliferation and migration and invasion in RCC cells [13]. However, the regulating effects between miR-154 and IncRNAs have never explored before.

In our present study, we noticed the down-regulation of LINC01939 in RCC tissues. Further study revealed that overexpressed LINC01939 suppressed proliferation and migration and promoted apoptosis by downregulating miR-154 with bluntness of Wnt/ $\beta$-catenin and Notch, providing novel diagnostic and therapeutic targets for RCC.

\section{Materials And Methods}

\section{Clinical specimens}

Kidney cancer tissue specimens were acquired from patients $(n=18)$ who had tumor resection at the First Affiliated Hospital of Soochow University. Para-carcinoma tissues were obtained from the resected tissues. Prior written and informed consents were obtained from all volunteer patients. The study was approved by the Medical Ethics Committee of the First Affiliated Hospital of Soochow University, and the process was performed in accordance with the guidelines.

\section{Cell culture}

Human renal cell adenocarcinoma cell line ACHN was obtained from Cell Lines Service (Item No. 300117; Eppelheim, Germany). ACHN cells were grown in Eagle's minimum essential medium with addition of fetal bovine serum (FBS) (Sigma-Aldrich, St Louis, MO, USA) to a final concentration of $10 \%$, and the cells were cultured at $37^{\circ} \mathrm{C}$ in atmosphere of $95 \%$ air, $5 \% \mathrm{CO}_{2}$. Human kidney carcinoma cell line CAKI- 1 was provided by DSMZ (Item No. ACC731; Braunschweig, Germany). CAKI-1 cells were seeded in McCoy's 5a medium (Gibco, Gaithersburg, MD, USA) in supplementation with $20 \% \mathrm{FBS}$, and the cells were cultured in the atmosphere of $95 \%$ air and $5 \% \mathrm{CO}_{2}$ at $37^{\circ} \mathrm{C}$.

\section{Cell transfection}


The full-length of LINC01939 sequences were constructed in pcDNA3.1 plasmid (Invitrogen, Carlsbad, CA, USA) to construct the recombinant plasmid pcDNA3.1-LINC01939. ACHN and CAKI-1 cells transfected with the empty vector served as the control. Following the manufacturer's instructions, pcDNA3.1LINC01939 or empty plasmid was introduced into ACHN and CAKI-1 cells by using lipofectamine 3000 reagent (Life Technologies, Carlsbad, CA, USA). Subsequently, the cells were grown in the culture medium containing $0.5 \mathrm{mg} / \mathrm{ml} \mathrm{G} 418$ (Sigma-Aldrich). Synthesized by Life Technologies, miR-154 mimic and the negative control mimic were transfected into ACHN and CAKI-1 cells. The expression of LINC01939 and miR-154 was quantified by quantitative real-time polymerase chain reaction (qRT-PCR).

\section{Cell viability}

To detect the viable cells, ACHN and CAKI- 1 cells (5,000 cells per well) were inoculated in 96-well plates, respectively. The cells were pre-incubated for $24 \mathrm{~h}$. Next, $10 \mu \mathrm{l}$ of CCK-8 solution (APExBIO, Houston, TX, USA) was added into each well. After co-incubation for $1 \mathrm{~h}$, the absorbance was recorded using Elx800 Reader (Bio-Tek, Winooski, VT, USA) at $450 \mathrm{~nm}$.

\section{Apoptosis assay}

The apoptotic cells were observed after stained in Annexin V-FITC/propidium iodide (PI) (Biosea, Beijing, China) under FACS Calibur Flow Cytometer (BD Biosciences, San Jose, CA, USA). In short, ACHN and CAKI- 1 cells were collected and digested with trypsin (Pierce, Appleton, WI, USA). After washed in pre-cold phosphate buffered saline (Sigma-Aldrich), the cells were collected by centrifugation. Then $5 \mu$ l of PI was added and co-incubated with the culture for $30 \mathrm{~min}$. The apoptotic cells were analyzed at $488 \mathrm{~nm}$.

\section{Western Blot}

The CAKI-1 and ACHN cells were lysed using RIPA lysis buffer (Beyotime, Shanghai, China). The extracted protein content was quantified using the $\mathrm{BCA}^{\mathrm{TM}}$ Protein Assay Kit (Pierce). After separation by sodium dodecyl sulfate polyacrylamide gel electrophoresis and transference onto polyvinylidene difluoride (PVDF) membrane (Millipore, Bedford, MA, USA), the proteins were blocked with $5 \%$ nonfat milk overnight at $4^{\circ} \mathrm{C}$. Next, the proteins were probed with primary antibodies at $4^{\circ} \mathrm{C}$ overnight. After washed, the membranes were incubated with secondary antibody for $1 \mathrm{~h}$ at room temperature. Finally, protein signaling was captured with an ECL system (Amersham Pharmacia, Piscataway, NJ, USA).

\section{Migration assay}

The migration activity of ACHN and CAKI-1 cells was valued by using a modified two-chamber (with a pore size of $8 \mathrm{~mm}$ ) migration assay. Firstly, the cells were plated in the top chamber in medium without 
serum, and the complete medium (600 $\mu \mathrm{l})$, FBS (with a final concentration of $10 \%)$ and epidermal growth factor $(25 \mathrm{ng} / \mathrm{ml}$ ) (Sigma-Aldrich) were supplemented into the lower compartment. After incubation for 24 h, non-traversed cells were removed from the upper surface of the filter. The cells on the lower surface were stained with crystal violet and counted.

\section{qRT-PCR}

Total RNA in RCC tissues and cell lines (ACHN and CAKI-1 cells) was isolated by using Trizol reagent (Invitrogen). MultiScribe RT Kit was used to perform reverse transcription reaction (Applied Biosystems, Foster City, CA, USA) with random hexamers or oligo(dT). PrimeScript RT Reagent Kit was exploited for PCR in the presence of SYBR Premix Ex Taq (TaKaRa, Dalian, China). Relative expression levels of LINC01939 or miR-154 were normalized against those of GAPDH or U6 snRNA and calculated by 2- $\Delta \Delta \mathrm{Ct}$ method.

\section{Statistical analysis}

SPSS 19.0 statistical software (Chicago, IL, USA) was applied to analyze the data which were computed as the mean \pm stand deviations (SD). Either one-way analysis of variance or student's $t$ test was used to compare the differences, and $P$-values less than 0.05 was accepted and suggested the statistical difference.

\section{Results}

\section{LINC01939 was down-regulated in renal carcinoma tissues}

The expression of LINC01939 in renal carcinoma tissues in the non-tumor tissues was firstly examined through qRT-PCR. As shown in Figure 1A (Fig.1A), the level of LINC01939 was suppressed in renal carcinoma tissues compared with the para-carcinoma tissues.

\section{Overexpression of LINC01939 facilitated apoptosis process in renal carcinoma cell lines}

Specific pc-LINC01939 was transduced into ACHN and CAKI-1 cells respectively to up-regulate the level of LINC01939 as shown in Fig.1B and the empty vector was used as a control. Then we found that cell viability was obviously suppressed in LINC01939-transfected (Fig.1C). Further, results from Western blotting assay showed that p53 was elevated and cyclinD1 was suppressed through transfecting with 
LINC01939 in both CAKI-1 and ACHN cells, identifying that cell proliferation was suppressed by overexpression of LINC01939 (Fig.1D). In addition, we found that LINC01939 overexpression obviously induced the apoptosis progress of ACHN and CAKI-1 cells (Fig.1E). At the same time, the expression of Bax and the cleavage of caspase-3 were also elevated by LINC01939 (Fig.1F). Thus, we concluded that overexpression of LINC01939 promoted the apoptosis of renal carcinoma cell lines.

\section{Migration activity of ACHN and CAKI-1 cells was weakened by LINC01939 overexpression}

The effect of overexpression of LINC01939 on the migration of renal carcinoma cell lines was further examined through wound healing assay and Western blot, respectively. The statistical results suggested that the migration behavior was largely suppressed by the overexpression of LINC01939 (Fig.2A). Besides, LINC01939 overexpression caused the abundance of MMP-2 and MMP-9, while the production of TIMP-1 was elevated by overexpressed LINC01939 (Fig.2B). Thus, we concluded that overexpression of LINC01939 suppressed the migration of renal carcinoma cell lines.

\section{LINC01939 overexpression-caused down-regulation of miR-154 functioned as a mediator to induce apoptosis}

Results from Fig.3A indicated that miR-154 was obviously suppressed by overexpressed LINC01939, suggesting that there may be a targeting relationship between LINC01939 and miR-154. In order to explore the connection between LINC01939 and miR-154, specific miR-154 mimic was used in our following experiments to elevate the accumulation of miR-154 as shown in Fig.3B. Then we observed that the viability inhibited by overexpressed LINC01939 was elevated by the combination of miR-154 mimic (Fig.3C). Besides, the inhibiting effect of LINC01939 on cell proliferation was also abolished by the adding of miR-154 mimic through suppressing the level of p53 and elevating the level of cyclin D1 (Fig.3D). In addition, elevated cell apoptosis rate by LINC01939 was then suppressed by overexpressed miR-154 (Fig.3E). At the same time, elevated expression of Bax and cleaved caspase-3 induced by LINC01939 was both suppressed by the combination of miR-154 mimic in CAKI- 1 and ACHN cells (Fig.3F). Thus, we conclude that overexpression of LINC01939 accelerated the apoptosis process by repressing the abundance of miR-154.

\section{LINC01939 overexpression impeded migration behavior by repressing the enrichment of miR-154}


The targeting relationship was also verified in cell migration. As shown in Fig.4A, overexpressed LINC01939 suppressed cell migration rate but the inhibiting effect was largely weakened by the combination of miR-154 mimic. At the same time, suppressed level of MMP-2 and MMP-9 was elevated and elevated expression of TIMP-1 was suppressed by the adding of miR-154 mimic compared with the effect of LINC01939 separately (Fig.4B). Thus, we concluded that overexpression of LINC01939 suppressed cell migration by down-regulating miR-154.

\section{Overexpression of LINC01939 retarded the activation of Wnt/ $\beta$-catenin and Notch signal pathways via down-regulating miR-154}

Related signal pathways were further investigated through Western blotting assay. As shown in Fig.5A-B, the expression of Wnt/ $\beta$-catenin signal pathway related proteins Wnt3a and $\beta$-catenin was suppressed by overexpressed LINC01939 and was then elevated by the combination of miR-154 mimic, indicating that overexpressed LINC01939 inactivated Wnt/ $\beta$-catenin via down-regulating miR-154. Similarly, the inhibiting effect of overexpressed LINC01939 on the Notch signal pathway was abolished by miR-154 mimic through elevating the expression of Notch1 and Notch2. Thus, we concluded that overexpression of LINC01939 inactivated Wnt/ $\beta$-catenin and Notch signal pathways via down-regulating miR-154.

\section{Discussion}

RCC originates from the renal parenchymal urinary tubular epithelial system, and it is widely accepted as a common malignant tumor in urinary system, accounting for nearly $2 \%-5 \%$ of adult malignant tumors worldwide [14]. With the advancement of imaging technology, many small RCC less than $3 \mathrm{~cm}$ were found, but many patients had already been in the advanced stage of the disease when they were found. Therefore, it is urgent to improve the diagnosis of RCC and to find new therapeutic targets in RCC treatment.

In recent years, IncRNAs have become a research hotspot and they are found to play a more and more important role in tumorigenesis and development. Studies have shown that IncRNAs play an important role in differentiation, proliferation processes, invasion and metastasis processes, and its abnormal expression causes the functional changes which might be a causal association with malignant tumors and other diseases [15]. Also, up to now, numerous IncRNAs have been found to participate in the tumorigenic and tumor-suppressive regulatory network in RCC. As an example, IncRNA termed metastatic renal cell carcinoma-associated transcript 1 (MRCCAT1) is ectopic up-regulated in metastatic ccRCC tissues, which might cause the metastatic capacity of ccRCC via mediating associated signaling transduction systems [16]. Besides, IncRNA UCA1 is overexpressed in CCRCC and the overexpression of UCA1 has been regarded as a poor prognostic biomarker in cCRCC diagnosis [17]. In our present study, we found LINC01939 was obviously down-regulated in RCC tissues. Further study found that 
overexpressed LINC01939 through exogenous transfection effectively repressed proliferation, accelerated apoptosis and blocked migration process of RCC cell lines. Consequently, we concluded that LINC01939 could be regarded as a novel diagnostic and therapeutic interest IncRNA for RCC.

miRNAs not only regulate the development and differentiation of cells, but also are potential molecular markers of tumors. They have good application prospects in early diagnosis, treatment, prognosis and chemotherapy resistance of tumors. MiRNAs act as a cancer-promoting or anti-cancer factor through epigenetic regulation and regulation of the expression of processing genes and proteins [ 18,19$]$. For example, Wang et al. proved miR-154 is down-regulated in glioblastoma while miR-154 overexpression suppresses the growth of glioblastoma cells, which could act as a beneficial therapeutic target [20]. However, Lin et al. revealed that miR-154 is overexpressed in RCC specimens and cells, which exaggerates the growth of RCC cells [13]. Therefore, the same miRNA may play opposite roles in different tumors through different regulatory mechanisms.

Many IncRNAs have structures similar to the mRNA targeted by miRNAs, so miRNAs can negatively regulate IncRNA through mechanisms similar to those acting on mRNA. The regulatory mechanisms between IncRNA and microRNAs include the following types: IncRNA competes with 3'-UTR of target RNA of microRNAs to indirectly inhibit the negative regulation of target genes by miRNAs [21]; IncRNA act as competitive endogenous RNA and plays the role of molecular sponge of miRNAs to inhibit the expression of miRNAs [22]. In addition, IncRNA can indirectly regulate the expression of miRNAs through other proteins to regulate the progression of tumors. In our study, we found that LINC01939 overexpression suppressed miR-154 expression. Further study showed that miR-154 mimic abolished the inhibiting effect of LINC01939 on proliferation and migration and the promoting effect of LINC01939 on apoptosis. Thus, we concluded that overexpressed LINC01939 suppressed proliferation and migration and induced apoptosis dependent on the down-regulation of miR-154.

Wnt/ $\beta$-catenin and Notch signal pathways play important roles in kinds of cancers. Jin et al. reported that miR-135b stimulated the recurrence and metastasis of osteosarcoma via activating Wnt/ $\beta$-Catenin and Notch by multiple negative regulators [ 23]. In our present study, both of Wnt/ $\beta$-catenin and Notch signal pathways was inactivated by overexpressed LINC01939. However, the inhibiting role of LINC01939 overexpression on Wnt/ $\beta$-catenin and Notch was abolished by the combination of miR-154 mimic. Thus, we conclude that overexpressed LINC01939 inactivated Wnt/ $\beta$-catenin and Notch by suppressing miR154.

\section{Conclusion}

Taken together, we found that LINC01939 was down-regulated in RCC tissues. Overexpression of LINC01939 suppressed proliferation and migration and induced apoptosis by down-regulating miR-154. Further study also revealed that Wnt/ $\beta$-catenin and Notch were inactivated by overexpression of LINC01939 via suppressing miR-154. Our present study for the first time revealed the regulating effect 
and associated regulation mechanism of LINC01939 in RCC, providing novel diagnostic and therapeutic targets for RCC.

\section{Declarations}

\section{Ethics approval and consent to participate}

All procedures performed in studies involving human participants were in accordance with the ethical standards of the institutional committee and with the 1964 Helsinki declaration and its later amendments or comparable ethical standards. The research was encouraged by the Medical Ethics Committee of the First Affiliated Hospital of Soochow University.

\section{Consent for publication}

Not applicable.

\section{Availability of data and material}

The datasets used and/or analyzed during the current study are available from the corresponding author on reasonable request.

\section{Competing interests}

The authors declare that they have no conflict of interest.

\section{Funding}

This work was supported by National Natural Science Foundation of China $(81472401,81772708)$.

\section{Authors' contributions}

Conception and design: Rongyuan Zhang, Yuhua Huang, Jianquan Hou and Weijie Zhang; Administrative support: Rongyuan Zhang, Jianquan Hou and Weijie Zhang; Provision of study materials or patients: Rongyuan Zhang, Yuhua Huang, Baocai Xu and Chuan Lv; Collection and assembly of data: Rongyuan Zhang, Yuhua Huang, Chuan Lv and Weijie Zhang; Data analysis and interpretation: Rongyuan Zhang, Baocai Xu, Weijie Zhang and Jianquan Hou; Manuscript writing: All authors; Final approval of manuscript: All authors. 


\section{Acknowledgements}

None.

\section{References}

1. Siegel R, Ma J, Zou Z, Jemal A: Cancer statistics, 2014. CA: a cancer journal for clinicians 2014, 64(1):9-29.

2. Youssef YM, White NM, Grigull J, Krizova A, Samy C, Mejia-Guerrero S, Evans A, Yousef GM: Accurate molecular classification of kidney cancer subtypes using microRNA signature. European urology 2011, 59(5):721-730.

3. Gremel G, Djureinovic D, Niinivirta M, Laird A, Ljungqvist O, Johannesson H, Bergman J, Edqvist PH, Navani S, Khan $\mathrm{N}$ et al: A systematic search strategy identifies cubilin as independent prognostic marker for renal cell carcinoma. BMC cancer 2017, 17(1):9.

4. Wilusz JE, Sunwoo H, Spector DL: Long noncoding RNAs: functional surprises from the RNA world. Genes \& development 2009, 23(13):1494-1504.

5. Fatica A, Bozzoni I: Long non-coding RNAs: new players in cell differentiation and development. Nature reviews Genetics 2014, 15(1):7-21.

6. Hong Q, Li O, Zheng W, Xiao WZ, Zhang L, Wu D, Cai GY, He JC, Chen XM: LncRNA HOTAIR regulates HIF-1alpha/AXL signaling through inhibition of miR-217 in renal cell carcinoma. Cell death \& disease 2017, 8(5):e2772.

7. Lu Y, Liu WG, Lu JH, Liu ZJ, Li HB, Liu GJ, She HY, Li GY, Shi XH: LncRNA UCA1 promotes renal cell carcinoma proliferation through epigenetically repressing p21 expression and negatively regulating miR495. Tumour biology : the journal of the International Society for Oncodevelopmental Biology and Medicine 2017, 39(5):1010428317701632.

8. Chen S, Ma P, Li B, Zhu D, Chen X, Xiang Y, Wang T, Ren X, Liu C, Jin X: LncRNA CCAT1 inhibits cell apoptosis of renal cell carcinoma through up-regulation of Livin protein. Molecular and cellular biochemistry 2017, 434(1-2):135-142.

9. Chen CL, Ke Q, Luo M, Gao ZY, Li ZJ, Luo ZG, Liu DB: Loss of LINC01939 expression predicts progression and poor survival in gastric cancer. Pathology, research and practice 2018, 214(10):15391543.

10. Bartel DP: MicroRNAs: target recognition and regulatory functions. Cell 2009, 136(2):215-233.

11. Leucci E, Patella F, Waage J, Holmstrom K, Lindow M, Porse B, Kauppinen S, Lund AH: microRNA-9 targets the long non-coding RNA MALAT1 for degradation in the nucleus. Scientific reports 2013, 3:2535. 
12. Szabo Z, Szegedi K, Gombos K, Mahua C, Flasko T, Harda K, Halmos G: Expression of miRNA-21 and miRNA-221 in clear cell renal cell carcinoma (ccRCC) and their possible role in the development of ccRCC. Urologic oncology 2016, 34(12):533.e521-533.e527.

13. Lin C, Li Z, Chen P, Quan J, Pan X, Zhao L, Zhou L, Lai Y, He T, Xu W et al: Oncogene miR-154-5p regulates cellular function and acts as a molecular marker with poor prognosis in renal cell carcinoma. Life sciences 2018, 209:481-489.

14. Cheng SK, Chuah KL: Metastatic Renal Cell Carcinoma to the Pancreas: A Review. Archives of pathology \& laboratory medicine 2016, 140(6):598-602.

15. Gibb EA, Brown CJ, Lam WL: The functional role of long non-coding RNA in human carcinomas. Molecular cancer 2011, 10:38.

16. Li JK, Chen C, Liu JY, Shi JZ, Liu SP, Liu B, Wu DS, Fang ZY, Bao Y, Jiang MM et al: Long noncoding RNA MRCCAT1 promotes metastasis of clear cell renal cell carcinoma via inhibiting NPR3 and activating p38-MAPK signaling. Molecular cancer 2017, 16(1):111.

17. Wang Y, Gao W, Xu J, Zhu Y, Liu L: The long noncoding RNA urothelial carcinoma-associated 1 overexpression as a poor prognostic biomarker in clear cell renal cell carcinoma. Tumour biology : the journal of the International Society for Oncodevelopmental Biology and Medicine 2017, 39(5):1010428317698377.

18. Erdmann K, Kaulke K, Thomae C, Huebner D, Sergon M, Froehner M, Wirth MP, Fuessel S: Elevated expression of prostate cancer-associated genes is linked to down-regulation of microRNAs. BMC cancer $2014,14: 82$.

19. Mulrane L, Klinger R, McGee SF, Gallagher WM, O'Connor DP: microRNAs: a new class of breast cancer biomarkers. Expert review of molecular diagnostics 2014, 14(3):347-363.

20. Wang X, Sun S, Tong X, Ma Q, Di H, Fu T, Sun Z, Cai Y, Fan W, Wu Q et al: MiRNA-154-5p inhibits cell proliferation and metastasis by targeting PIWIL1 in glioblastoma. Brain research 2017, 1676:69-76.

21. Faghihi MA, Modarresi F, Khalil AM, Wood DE, Sahagan BG, Morgan TE, Finch CE, St Laurent G, 3rd, Kenny PJ, Wahlestedt C: Expression of a noncoding RNA is elevated in Alzheimer's disease and drives rapid feed-forward regulation of beta-secretase. Nature medicine 2008, 14(7):723-730.

22. Salmena L, Poliseno L, Tay Y, Kats L, Pandolfi PP: A ceRNA hypothesis: the Rosetta Stone of a hidden RNA language? Cel/2011, 146(3):353-358.

23. Jin H, Luo S, Wang Y, Liu C, Piao Z, Xu M, Guan W, Li Q, Zou H, Tan QY et al: miR-135b Stimulates Osteosarcoma Recurrence and Lung Metastasis via Notch and Wnt/beta-Catenin Signaling. Molecular therapy Nucleic acids 2017, 8:111-122. 
Figures
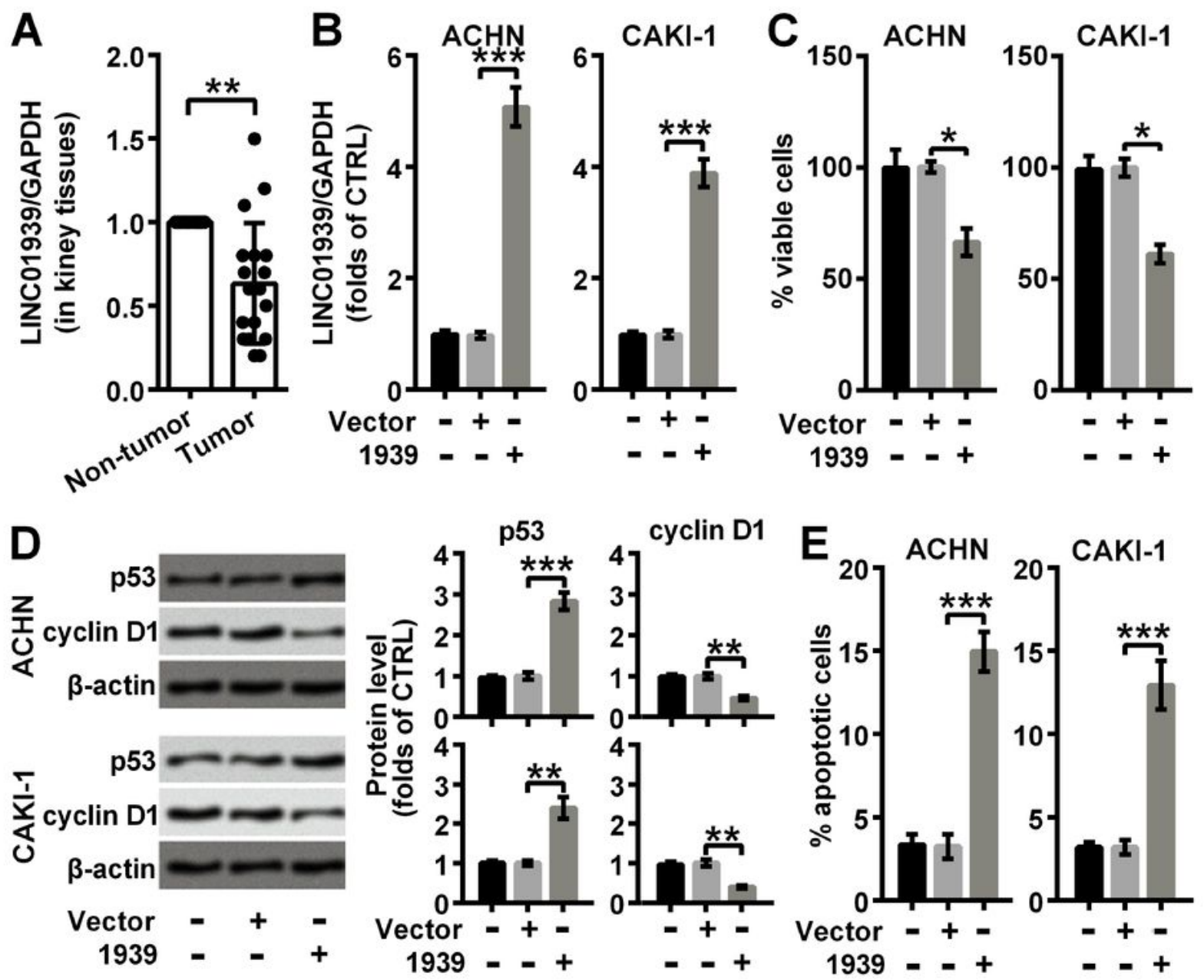

CAKI-1

$\mathbf{F}$

종 $\mathrm{Bax}$

$\beta$-actin

Bax
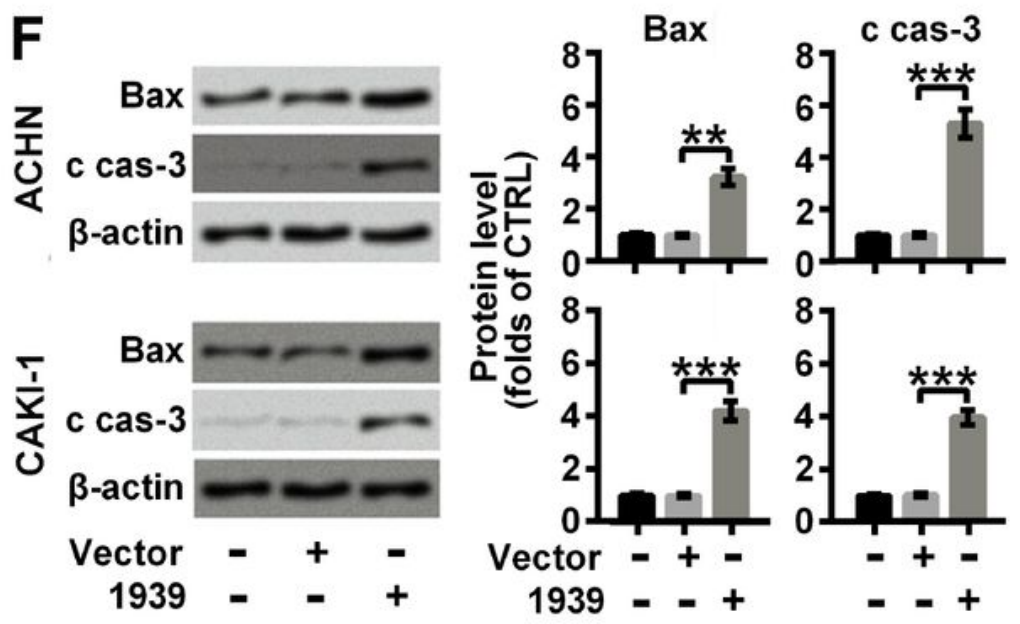

Figure 1

LINC01939 was lowly expressed in renal carcinoma tissues and LINC01939 overexpression exhibited a pro-apoptosis role in ACHN and CAKI-1 cells. A. The expression of LINC01939 in renal carcinoma tissues and para-carcinoma tissues was examined through qRT-PCR. ACHN and CAKI- 1 cells were transfected 
with the empty vector pcDNA3.1 (Vector) or pcDNA3.1-LINC01939 (1939) as indicated. B. LINC01939 was analyzed through qRT-PCR. C. Viability of cells was valued through CCK8 assay. D. Western blotting assay of proliferation related proteins p53 and cyclin D1 was carried out. E. Apoptotic cells was examined through flow cytometry. F. Western blots of apoptosis related proteins Bax and cleaved caspase-3 (c cas-

3). Quantifications were expressed as means of three independent experiments $\pm S D$. ${ }^{*} P<0.05$, ${ }^{*} P<$ $0.01, * \star * P<0.001$.
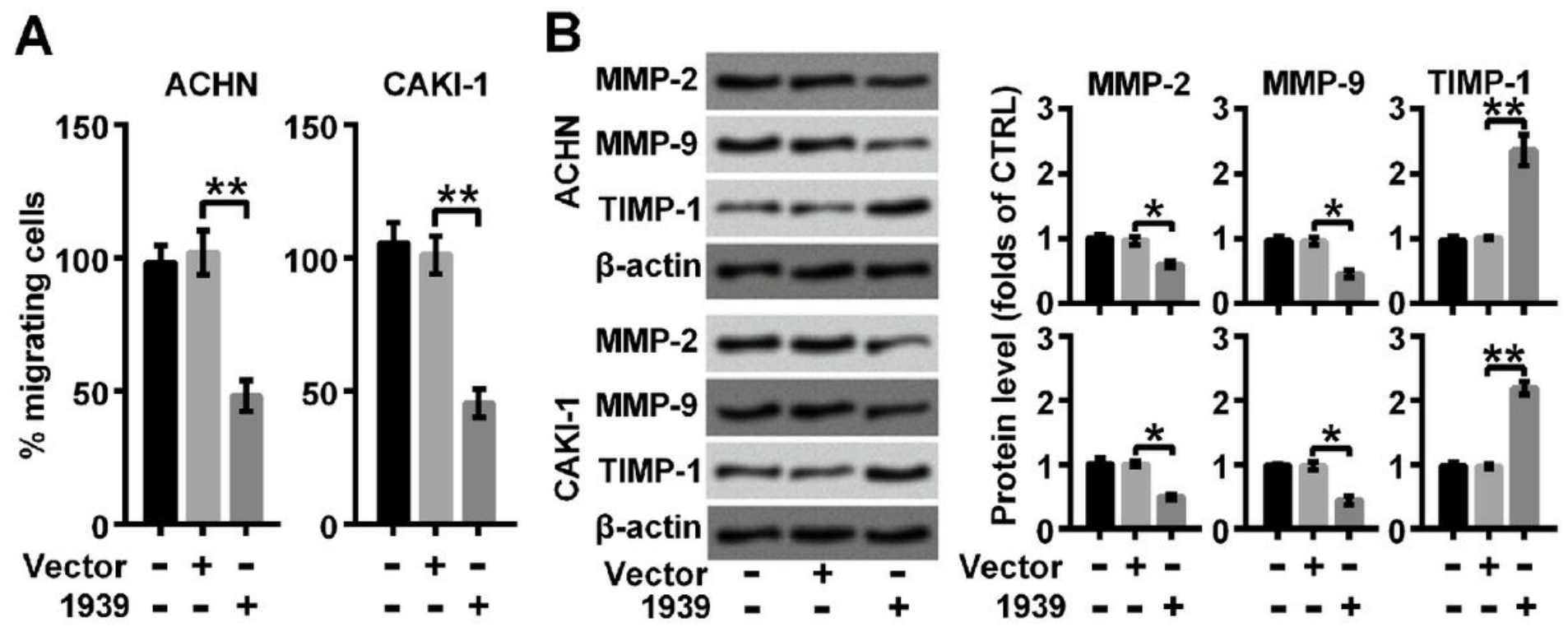

Figure 2

LINC01939 overexpression suppressed migration activity of ACHN and CAKI-1 cells. ACHN and CAKI-1 cells were transfected with the empty vector pcDNA3.1 (Vector) or pcDNA3.1-LINC01939 (1939) as indicated. A. The migration behavior was evaluated by wound healing assay. B. Western blotting assay of migration related proteins matrix metallopeptidase (MMP)-2, MMP-9, and TIMP metallopeptidase inhibitor 1 (TIMP-1) was performed. Quantifications were expressed as means of three independent experiments $\pm S D$. $* P<0.05$ and ${ }^{* *} \mathrm{P}<0.01$. 

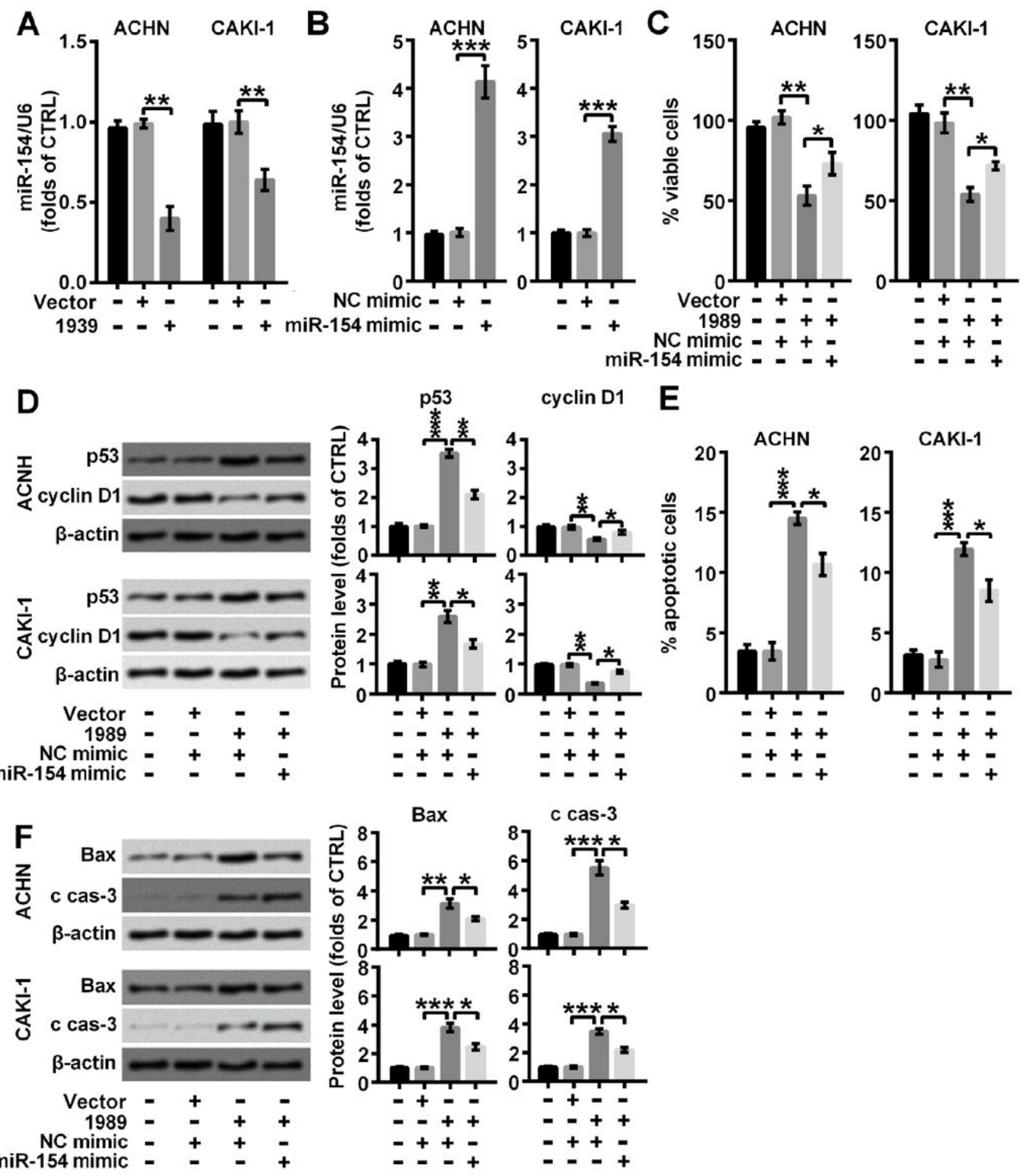

Figure 3

LINC01939 promoted apoptosis process via controlling the abundance of miR-154. A. ACHN and CAKI-1 cells were transfected with the empty vector pcDNA3.1 (Vector) or pcDNA3.1-LINC01939 (1939) as indicated. miR-154 was examined through qRT-PCR. B. ACHN and CAKI- 1 cells were transfected with NC (negative control) mimic or miR-154 mimic as indicated. The expression of miR-154 was examined through qRT-PCR. ACHN and CAKI-1 cells were transfected with pcDNA3.1-LINC01939 and miR-154 
mimic. C. Viability was valued through CCK8 assay. D. Western blotting assay of proliferation related proteins p53 and cyclin D1 was carried out. E. Apoptotic cells were examined through flow cytometry. F. Western blotting assay of apoptosis related proteins Bax and cleaved caspase-3 (c cas-3).

Quantifications were expressed as means of three independent experiments \pm SD. ${ }^{*} P<0.05,{ }^{\star *} P<0.01$, $\star \star * P<0.001$.
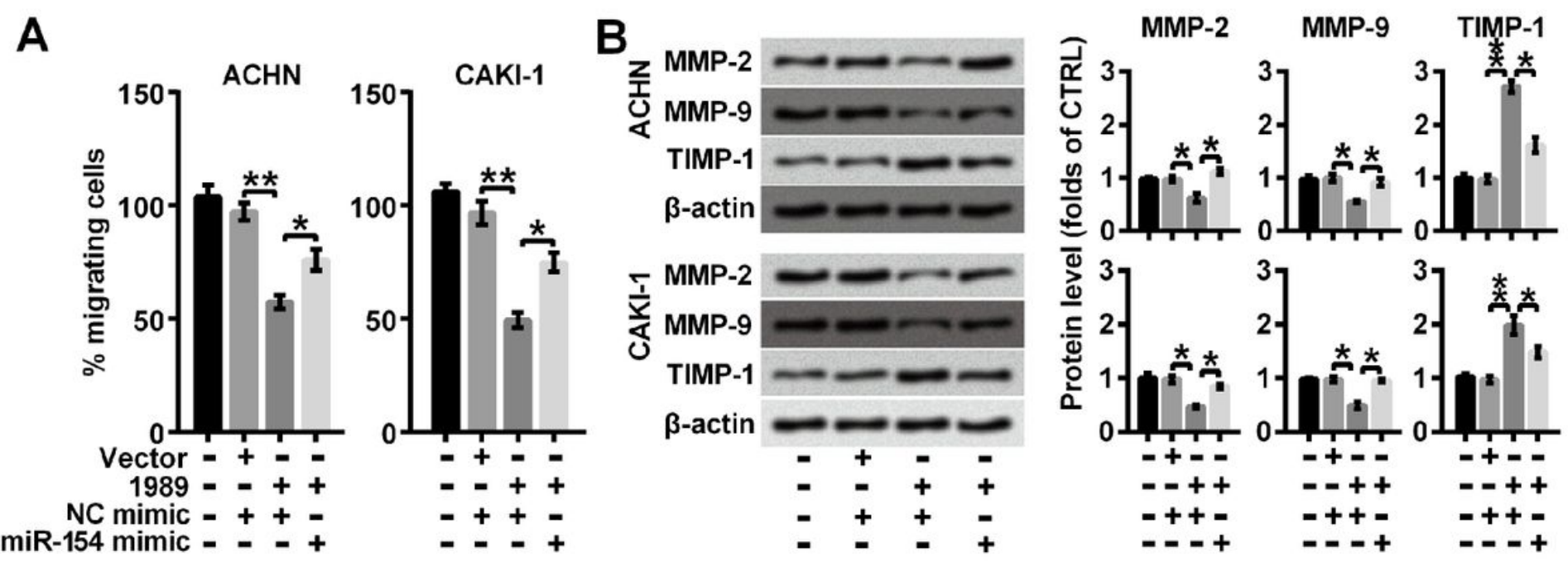

Figure 4

LINC01939 overexpression suppressed migration via up-regulating miR-154. ACHN and CAKI-1 cells were transfected with NC (negative control) mimic or miR-154 mimic as indicated. A. The migration behavior was evaluated by wound healing assay. B. Western blotting assay of migration related proteins matrix metallopeptidase (MMP)-2, MMP-9, and TIMP metallopeptidase inhibitor 1 (TIMP-1) was performed. Quantifications were expressed as means of three independent experiments $\pm S D$. ${ }^{\star} P<0.05,{ }^{\star} * P<0.01$. 
A
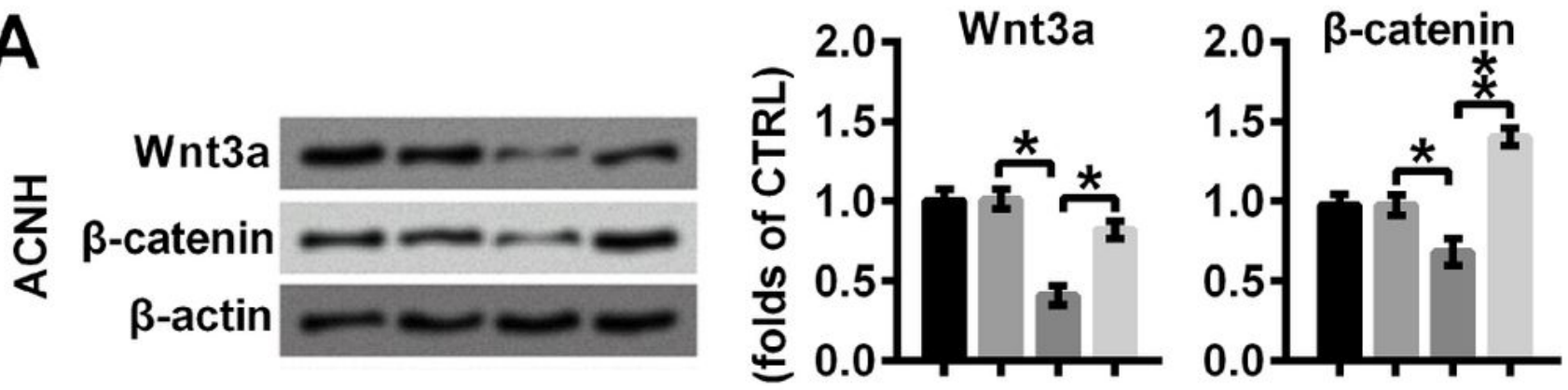

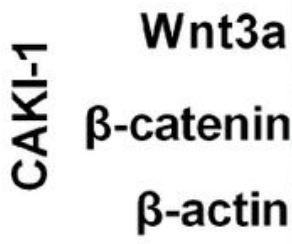
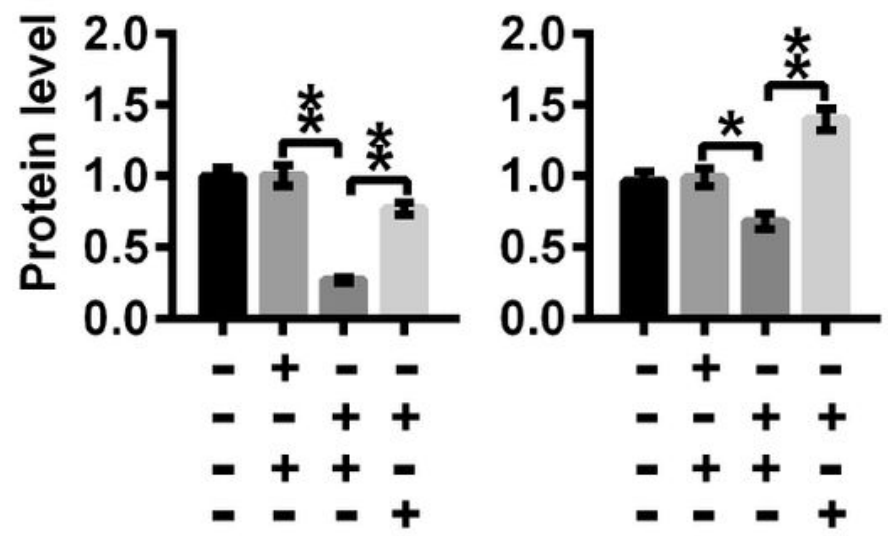

miR-154 mimic $= \pm+ \pm+$
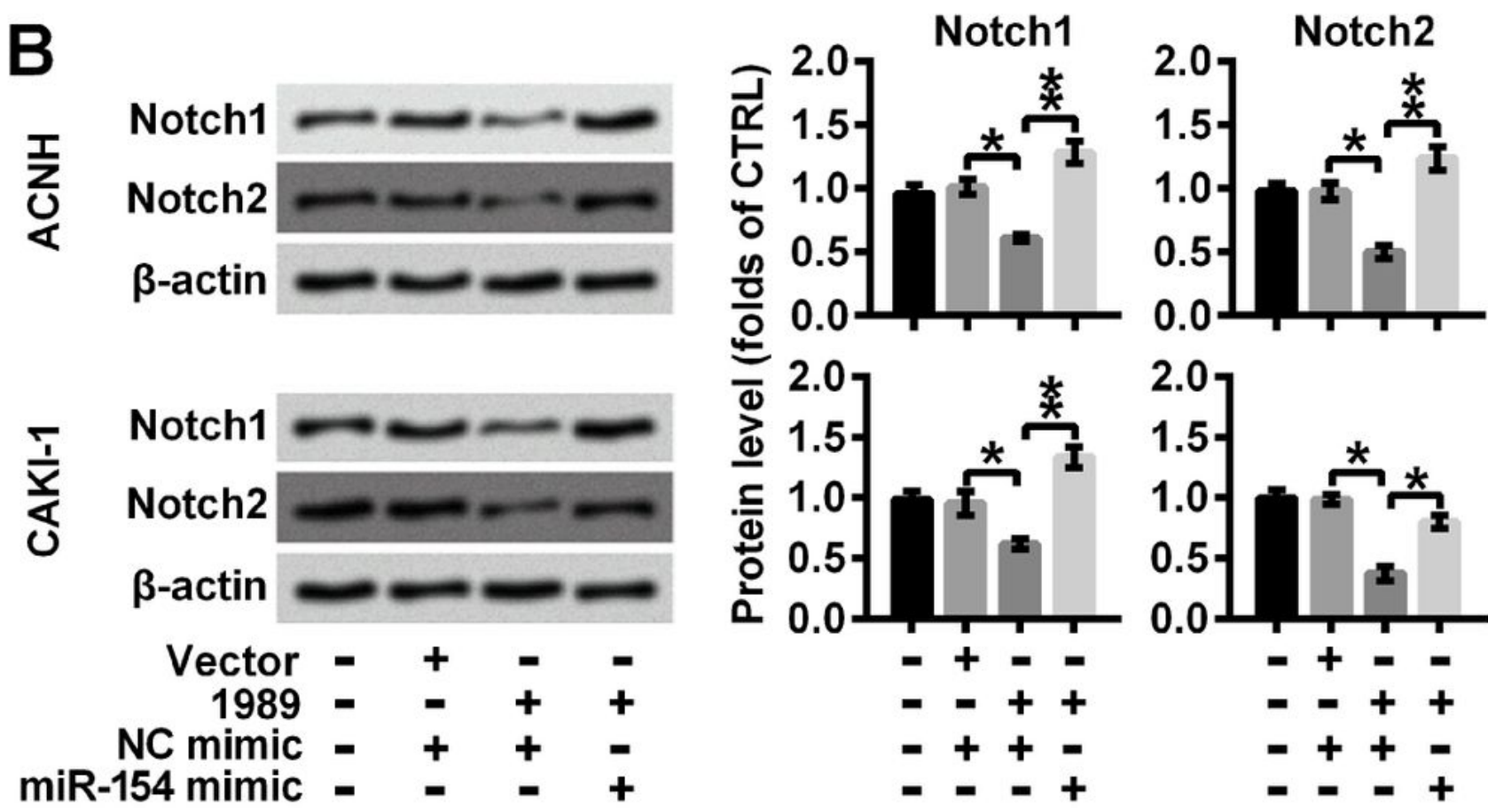

Figure 5

LINC01939 overexpression-caused blockade of Wnt3a/ $\beta$-catenin and Notch might be mediated by the down-regulation of miR-154. ACHN and CAKI-1 cells were transfected with NC (negative control) mimic or miR-154 mimic as indicated. Western blotting assay of signaling mediators of $(A)$ Wnt3a/ $\beta$-catenin (Wnt3a and $\beta$-catenin) and (B) Notch (Notch1 and Notch2) pathways was performed. Quantifications were expressed as means of three independent experiments \pm SD. ${ }^{*} \mathrm{P}<0.05, * * \mathrm{P}<0.01$. 